\title{
Dynamic ANN for precipitation estimation and forecasting from radar observations
}

\author{
Yen-Ming Chiang ${ }^{a}$, Fi-John Chang ${ }^{a, *}$, Ben Jong-Dao Jou ${ }^{b}$, Pin-Fang Lin ${ }^{b}$
}

\author{
a Department of Bioenvironmental Systems Engineering, National Taiwan University, No. 1, Sec. 4, \\ Roosevelt Road, Taipei, Taiwan, ROC \\ b Department of Atmospheric Sciences, National Taiwan University, Taipei, Taiwan, ROC
}

Received 3 November 2005; received in revised form 12 October 2006; accepted 12 October 2006

\section{KEYWORDS \\ Quantitative precipita- tion forecasting; \\ Dynamic artificial neural network; \\ $\mathbf{Z}-\mathbf{R}$ relation; \\ Radar measurements}

\begin{abstract}
Summary Instantaneous quantitative precipitation estimation (QPE) and quantitative precipitation forecasting (QPF) by utilizing the meteorological radar data are potentially of great benefit for operational hydrology in river basins. The most commonly used technique of radar-based rainfall estimation is a best fitted power-law function between reflectivity $(Z)$ and rain intensity $(\boldsymbol{R})$. An emerging tool in QPE/QPF using radar data is the artificial neural network (ANN) that is capable of learning complex nonlinear relationships. In this study, we introduce a dynamic ANN approach to construct instantaneous QPE and one-hour-ahead QPF by using a three-dimensional radar data structure, which takes into account the terminal velocity and the horizontal advection. Radar measurements and three rain gauges for six typhoon events in the Keelung River, Taiwan, were used for calibrating and evaluating the $Z-\boldsymbol{R}$ relation and dynamic ANN models. The results of current rainfall estimation and one-hour-ahead rainfall forecasting all indicate that the dynamic ANN can produce much more accurate and stable performance than the $\mathbf{Z}-\boldsymbol{R}$ power-law models. This study demonstrates that the dynamic ANN can be applied successfully in instantaneous QPE and QPF by using remote sensing data.

(C) 2006 Elsevier B.V. All rights reserved.
\end{abstract}

\section{Introduction}

Water resource in the form of rainfall is a valuable product and required for the protection of human life. Large-scale variations of precipitation lead to droughts and floods,

\footnotetext{
* Corresponding author. Tel.: +886 2 23639461; fax: +886 2 23635854.

E-mail address: changfj@ntu.edu.tw (F.-J. Chang).
}

two very important hydrological phenomena in terms of their impact on the ecosystem. Therefore, quantitative precipitation estimation/forecasting (QPE/QPF) provides essential information for practical applications in various fields such as reservoir operation, water resources management, agriculture, and flood protection. Taiwan has high mountains and a subtropical climate where typhoons, usually coupled with heavy rainfall, hit the island more than three times a year on average, causing downstream flooding 
within a few hours. Consequently, hourly rainfall estimations are quite useful and essential for flood monitoring and/or forecasting.

Traditionally, rainfall is measured by ground rain gauges, a simple and convenient method, but their spatial representations are exceedingly poor. Since the introduction of remotely sensed information in operational hydrological forecasting several decades ago, a multisensor algorithm that uses radar/satellite and rain gauge measurements has been widely acknowledged as one of the most important and promising procedures in hydrometeorology (Grecu and Anagnostou, 2001; Gourley et al., 2002; Seo and Breidenbach, 2002; Kidd et al., 2003). Rainfall estimation from radar observations is an attractive tool as it has better resolution than satellite data and is available in real time. The finer temporal and spatial resolution of radar observations as compared with rain gauges allows inclusion of high-intensity, isolated cells that may not be captured by rain gauges. The advantage of radar measurement is that the rainfall of a large area can be established through the high spatial and temporal resolution of radar data. Besides, with a radar network, the three dimensional structure of rainfall systems can be extended over a large area by compositing data from several radars. Since weather radar has been employed, a series of studies (Baltas and Mimikou, 1994; Grecu and Krajewski, 2000; Morin et al., 2003) have raised issues regarding the accuracy of radar-based rainfall forecasting.

The most attractive capability of weather radars is their ability to measure rainfall rate using a relationship between radar echo power and rain intensity, that is, reflectivity factor $(Z)$ and rain rate $(R)$ relation. Both $Z$ and $R$ are defined as different moments of the drop size distribution (DSD) in a sampled volume (Sauvageot, 1992). The most common form of $Z$ and $R$ can be related as follows:

$Z=a R^{b}$

where $a$ and $b$ are empirical parameters.

The $Z-R$ relation was pioneered by Marshall and Palmer (1948); after that, many subsequent studies with numerous aspects were investigated and different values were suggested for the parameters. Nevertheless, there are many complex situations for which rainfall is highly variable both spatially and temporally and is associated with different rainfall patterns such as stratiform or convective types. Traditionally, the radar-based rainfall estimation has been calculated from the $Z-R$ relation, which may vary from place to place and season to season (Xiao and Chandrasekar, 1997). The applications of $Z-R$ relation are hence restricted within specific cases. It is obvious that an estimation technique based on this kind of relation will not be very accurate and/or successful since no single values for $a$ and $b$ could provide a good estimate of the rainfall over a broad range of conditions (Trafalis et al., 2002). In addition, both variables are subjected to uncertainty (Ciach and Krajewski, 1999; Morin et al., 2003); hence it is necessary to explore alternative approaches to reduce the variability between reflectivity and rain rate.

The artificial neural network (ANN) is one of the most prominent and novel ways in hydrology for finding rainfall characteristics. ANNs are complex data processing methods that have been shown to act as function approximations.
The main advantages of using ANNs are that they are effective in extracting significant features and trends from complex data structures, capable of performing any nonlinear input-output mapping, and robust or error tolerant, which implies that error at a few input nodes or links will not significantly influence the overall performance. Recently, the use of ANN to combine remote sensing and rain gauge data has been highlighted. In the field of rainfall forecasting, many studies have been performed using an ANN approach with different remote sensing data such as satellite data (Hsu et al., 1997; Sorooshian et al., 2000; Kuligowski and Barros, 2001; Grimes et al., 2003) and radar data (Mimikou and Baltas, 1996; Bellerby et al., 2000; Trafalis et al., 2002). All of these studies have reported an improvement in performance by using ANNs. For example, an adaptive neural network was implemented to estimate rainfall rates using infrared satellite imagery and ground-based rain gauges (Hsu et al., 1997). A neural network with principal component analysis to rainfall estimation on a daily timescale in African river basin was proposed by Grimes et al. (2003). Trafalis et al. (2002) introduced data mining and an intelligent system to improve WSR-88D rainfall estimation.

The main purpose of this study is to use a recurrent neural network (RNN) for effective and accurate estimation and forecast the short-term (hourly) rainfall during typhoon periods. This will be accomplished by exploring and utilizing more fully the available types of radar data and build a dynamic nonlinear relation between radar measurements and ground rain gauge data. In order to learn the complex relationship of rainfall at ground gages and radar measurements, we construct the instantaneous QPE and QPF models by using an ANN and three-dimensional radar observations. The organization of this paper is as follows. "Data sources and processing" describes the study area and the processing of radar data. In "QPE and QPF using recurrent neural network", the methodology of constructing the QPE and QPF are presented, which includes an overview, the radar and rain gauge data used, and the architectures of the instantaneous QPE and QPF models. A comparison of the results obtained by RNN and $Z-R$ relation is shown in "Results and discussion". We close with concluding remarks in "Concluding remarks".

\section{Data sources and processing}

\section{Study area}

This section describes the study area and provides an overview of the data used. Radar and rain gauge observations collected within the study region were used to construct and examine the neural network-based QPE and QPF models. The Keelung River was selected as our study area, which is one of the chief tributaries of the Tanshui River located in northern Taiwan surrounding the metropolis of Taipei with a drainage area of approximately $500 \mathrm{~km}^{2}$. Locations of the Keelung River basin, rain gauges and radar station are shown in Fig. 1, where three rain gauges and the radar are denoted by circles and a triangle, respectively. The watershed is located $45-65 \mathrm{~km}$ east of the radar station. At this distance, attenuation caused by the atmosphere is usually quite small 


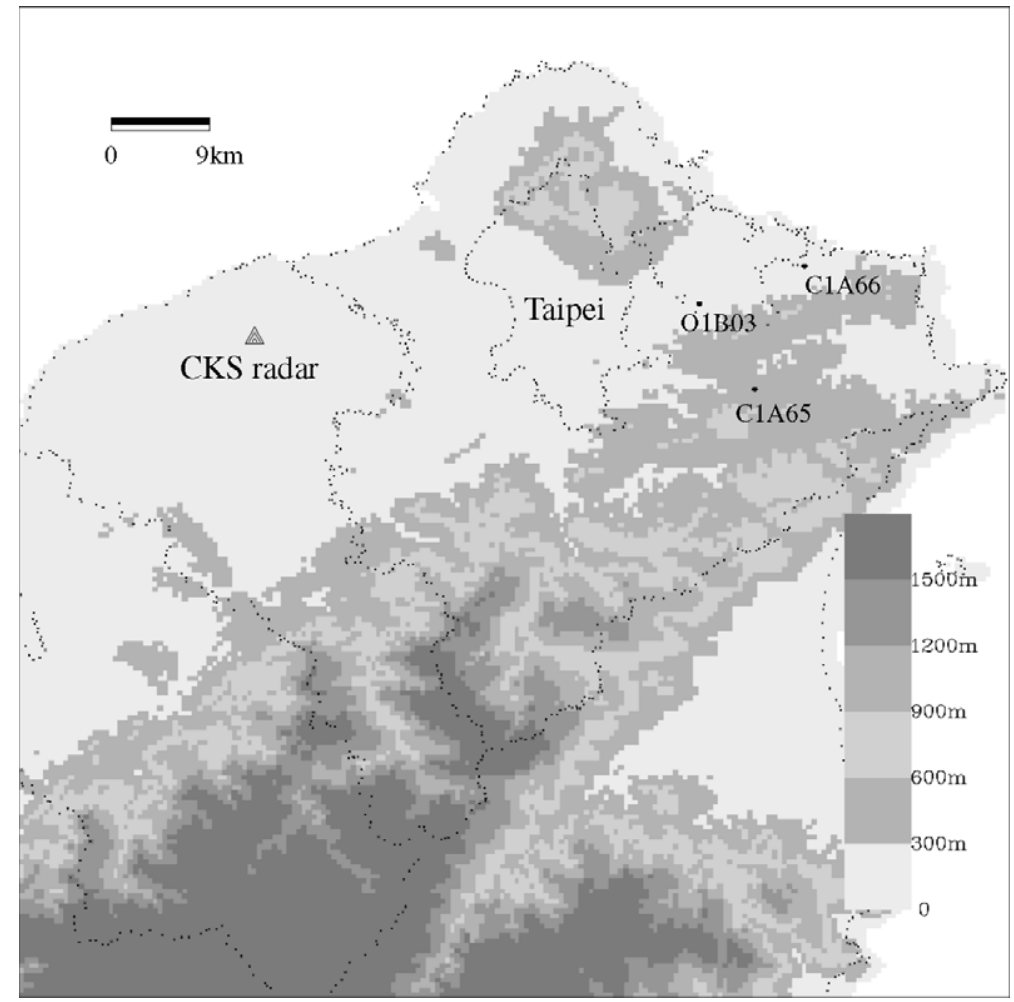

Figure 1 Location of study area, rain gauges, and radar stations.

and can often be neglected. Besides, an inspection of Fig. 1 shows that the influence of terrain on the lowest elevation scan is also insignificant. Rainfall measurements from the ground gauges located at 01B03, C1A65, and C1A66 with a recording resolution of $1 \mathrm{~h}$ were obtained from the Water Resources Agency, Taiwan. A series of typhoon events data were chosen and processed. The hourly data from these rain gauges were collected during the period 1990-1997 and 2004 consisting six typhoon events: Yancy (9012), Ruth (9123), Ted (9219), Herb (9608), Winnie (9714), and Nanmadol (0427). Simultaneous radar data were also collected and processed to provide instantaneous QPE and QPF by the proposed methods.

\section{Processing of radar data}

The radar data used in the analysis were taken from a Cband Doppler radar at Chiang Kai-Shek (CKS) international airport that has an effective range of $120 \mathrm{~km}$ with Doppler mode. Table 1 lists the technical characteristics of CKS radar that are relevant to this study. The radar performs approximately 10 different elevation scans that consist of a complete volume scan. Each elevation scan is at a particular angle between $0.5^{\circ}$ and $15^{\circ}$ above the horizontal. For each elevation scan, the radar revolves a full $360^{\circ}$ and makes about 420 azimuthal scans in approximately $25 \mathrm{~s}$. In other words, the radar completes a volume scan in about $4.2 \mathrm{~min}$. Even though the radar can produce a full scan every $4.2 \mathrm{~min}$, it is not operating continuously in order to reduce the data load. The CKS radar usually completes one volume scan per hour, except during a typhoon period when it completes two volume scans every hour. Hence, the temporal resolution of the volume scan is either half an hour or one hour. The radar parameter of interest to this research is the reflectivity factor at horizontal polarization and is recorded once in every kilometer for each azimuthal scan. The data processing steps of the CKS radar are as follows for each single elevation scan (polar coordinates).

Table 1 The technical characteristics of the CKS radar

\begin{tabular}{|c|c|c|}
\hline Type & \multicolumn{2}{|l|}{ USA421 } \\
\hline Location & \multicolumn{2}{|c|}{$121.20^{\circ} \mathrm{E} ; 25.07^{\circ} \mathrm{N}$} \\
\hline Altitude & \multicolumn{2}{|l|}{$27 \mathrm{~m}$} \\
\hline Antenna diameter & \multicolumn{2}{|l|}{$4.2 \mathrm{~m}$} \\
\hline Beamwidth & \multicolumn{2}{|l|}{$0.857^{\circ}$} \\
\hline Antenna gain & \multicolumn{2}{|l|}{$43 \mathrm{~dB}$} \\
\hline Side lobe & \multicolumn{2}{|l|}{$-27 \mathrm{~dB}$} \\
\hline Antenna polarization & \multicolumn{2}{|c|}{ Horizontal } \\
\hline Peak power & \multicolumn{2}{|c|}{$250 \mathrm{~kW}$} \\
\hline Noise figure & \multicolumn{2}{|l|}{$5 \mathrm{~dB}$} \\
\hline & Non-doppler & doppler \\
\hline Maximum rotation rate (RPM) & $2,4,6$ & 2,4 \\
\hline Pulse duration $(\mu \mathrm{s})$ & 2.0 & 0.5 \\
\hline Pulse repetition frequency $(\mathrm{Hz})$ & 250 & $900 / 1200$ \\
\hline Receiver & Logarithmic & Linear \\
\hline Number of samples (pulses) & 32 & 64 \\
\hline Clutter suppression $(\mathrm{dB})$ & $>32$ & $>26$ \\
\hline Range coverage (km) & 240 & 120 \\
\hline Range resolution (km) & 2 & 1 \\
\hline
\end{tabular}


1. Reflectivity data smaller than $0 \mathrm{dBZ}$ are eliminated.

2. By searching in a $5 \times 5$-pixel window surrounding a given bin. If there are less than two valid data points, then the central data point is deleted.

Because the raw radar data are originally arranged in polar coordinates, the raw data are converted to Cartesian coordinates in $\mathrm{dBZ}$ with grid dimensions $1 \times 1 \mathrm{~km}^{2}$. Meanwhile, missing data are interpolated by using a Cressman weighting function (Miller et al., 1986) as shown below. In this study, the radius used in the directions of $X, Y$ and $Z$ are $1.5 \mathrm{~km}, 1.5 \mathrm{~km}$ and $1 \mathrm{~km}$, respectively.

$R^{2}=\mathrm{d} X^{2}+\mathrm{d} Y^{2}+\mathrm{d} Z^{2}$

$W(r)= \begin{cases}\frac{R^{2}-r^{2}}{R^{2}+r^{2}}, & r \leqslant R \\ 0, & r>R\end{cases}$

where $R$ is the radius of influence and $r$ is the distance between the gate and the grid point.

In order to provide a consistent format for the input data to the neural network, we generated constant altitude plan position indicators (CAPPI) at different altitudes that are centered on each rain gauge location. It should be noted that the temporal resolution of rain gauge data used in this study is one hour, whereas the temporal resolution of radar data used herein is either half an hour or one hour, which means the time interval of radar data is irregular. Therefore, the time period should be the same order as the rain gauge time. One way to solve the time order problem is using the hourly reflectivity data. The scaled reflectivity factor with temporal resolution of $1 \mathrm{~h}$ were then obtained from two volume scans that were linearly interpolated to match the gauge recording time. After data processing, both rain gauge and radar data have a resolution of $1 \mathrm{~h}$. We have arbitrarily selected four typhoon events for calibration, one event for first evaluation (typhoon Herb), and one recent event for second evaluation (typhoon Nanmadol). Table 2 shows the collected events used in this study.

\section{QPE and QPF using recurrent neural network}

The ability of the brain to perform difficult operations, to recognize complex patterns and to learn from experience without a priori knowledge makes it an extraordinarily flexible and powerful computing tool. The ANN is a massively parallel computing system for which original development was inspired by the high efficiency of the brain. The attractiveness of ANN comes from information processing charac- teristics such as non-linearity, noise tolerance, and generalization capability. An ANN usually consists of three layers namely input, hidden, and output layer. The layers are connected by weights. Several types of structures were investigated since the development of ANN. However, we can easily distinguish them into two different generic neural network types if we take the network topologies into account, that is, feedforward and feedback networks.

The feedforward neural network was used to deal with fixed-weights mapping from input layer to output layer and it produced satisfactory performance in a static system (Ham and Kostanic, 2001). Because of the fixed weights, the state of each output neuron is only determined by the current input neuron and not the initial or past states of the neurons in the network; consequently, they often can not well cope with a dynamic or time-varying system. To allow initial and past state involvement along with serial processing, the RNN utilizes feedback links. The feedback links in the RNN can be interpreted as an extra context-layer, which store relevant temporal information or replicate the hidden layer output signals at the previous time step. A network whose state varies with time can be regarded as a dynamic system which means that time constitutes an essential ingredient of the learning process. Therefore, the presence of feedback links has a profound impact on the learning capability of the network and on carrying out the sequential temporal behavior. Chiang et al. (2004) made a series of systematic comparisons between feedforward and feedback architecture and demonstrated that RNN generally could produce better and more stable performance than feedforward neural network. The network architecture used in this paper is the RNN that has feedback connections originating from the outputs of the hidden layer to its input layer, and its learning algorithm is briefly summarized as follows.

\section{Real-time recurrent learning algorithm}

The learning algorithm that was used to calibrate the RNN is referred to as real-time recurrent learning (RTRL). The algorithm derives its name from the fact that adjustments are made to the synaptic weights of a fully connected RNN in a real-time manner, that is, the network continues to perform (adjust) its signal processing function (Williams and Zipser, 1989). This feature is especially important and useful for grasping the extraordinary time- and spatial-varying characteristics of rainfall processes. Consider a three-layer fully interconnected RNN as shown in Fig. 2, which includes $M$ external inputs, $N$ hidden neurons and $K$ outputs. Let $V$ and $W$ denote the $N \times K$ weight matrix and $N \times(M+N)$ recur-

Table 2 The typhoon events used in this study

\begin{tabular}{lllll}
\hline Events & Name and no. & Configuration & Duration & Maximum rainfall $(\mathrm{mm})$ \\
\hline 1 & Yancy (9012) & Calibration & $1990 / 08 / 19$ & 75 \\
2 & Ruth (9123) & $1991 / 10 / 29 \sim 30$ & 38 \\
3 & Ted (9219) & $1992 / 09 / 21 \sim 22$ & 26 \\
4 & Winnie (9714) & $1997 / 08 / 17 \sim 18$ & 59 & 51 \\
5 & Herb (9608) & Evaluation \#1 & $1996 / 07 / 31 \sim 08 / 01$ & 65 \\
6 & Nanmadol (0427) & Evaluation \#2 & $2004 / 12 / 03 \sim 04$ & \\
\hline
\end{tabular}




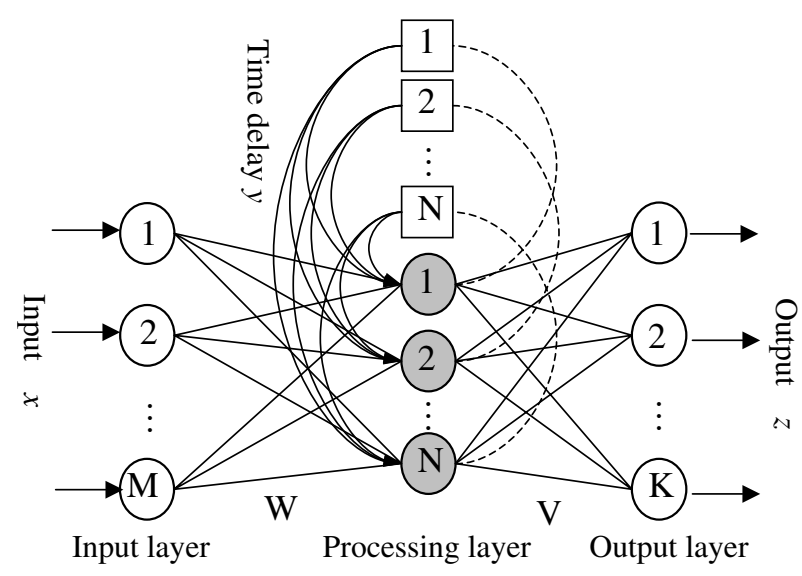

Figure 2 The architecture of a recurrent neural network.

rent weight matrix, respectively. The network activity of neuron $j$, for $j \in B$, is computed by

net $_{j}=\sum w_{j i} u_{i}$

where $u_{i}= \begin{cases}x_{i} & \text { if } i \in A \\ y_{i} & \text { if } i \in B\end{cases}$

$A$ denotes the set of indices $i$ for which $x_{i}$ is an external input, and $B$ denotes the set of indices $i$ for which $y_{i}$ is the output of the processing layer.

The output of neuron $j$ in the processing layer is given by passing net $_{j}$ through the nonlinear transfer function $f(\cdot)$, yielding

$y_{j}=f\left(\right.$ net $\left._{j}\right)$

The output of neuron $k$ in the output layer is computed by

net $_{k}=\sum v_{k j} y_{j}$

The output of network $\left(z_{k}\right)$ is given by passing net $t_{k}$ through the nonlinear transfer function $f(\cdot)$, yielding

$z_{k}=f\left(\right.$ net $\left._{k}\right)$

To minimize the objective function $\left(E_{\text {total }}\right)$, the steepest descent method is applied to adjust the weights ( $V$ and $W$ ) along the negative of $\nabla E_{\text {total }}$. The objective function is obtained by summing instantaneous network error $(E(t))$ over all time $T$ and $E(t)$ can be defined as follows:

$E_{\text {total }}=\sum_{t=1}^{T} E(t)$
$E(t)=\frac{1}{2} \sum_{k=1}^{K}\left[T_{k}(t)-z_{k}(t)\right]^{2}$

where $T_{k}(t)$ is the target value of neuron $k$ at time $t$, and $z_{k}(t)$ is the network output of neuron $k$ at time $t$.

The weight change for any particular weight $v_{k j}$ can thus be written as

$\Delta v_{k j}=-\eta_{1} \frac{\partial E}{\partial v_{k j}}$

By using the chain rule, the partial derivative of $v_{k j}$ can be obtained as follows:

$\Delta v_{k j}=\eta_{1} \sum_{k=1}^{K} e_{k} f^{\prime}\left(\right.$ net $\left._{k}\right) y_{j}$
The same method is also implemented for weight $w_{m n}$. Then the weight changes can be computed as

$\Delta w_{m n}=\eta_{2}\left(\sum_{k=1}^{K} e_{k} f^{\prime}\left(\right.\right.$ net $\left.\left._{k}\right) v_{k j}\right) \pi_{m n}^{j}$

where $\pi_{m n}^{j}(t+1)=f^{\prime}\left(\right.$ net $\left._{j}\right)\left(\sum_{i \in B} w_{j i} \pi_{m n}^{j}(t)+\delta_{m j} u_{n}\right) ; \eta_{1}$ and $\eta_{2}$ are the learning-rate parameters.

Detailed information on RTRL algorithm can be found in Chang et al. (2002, 2004).

\section{Description of QPE and QPF models}

We used the RNN-based approach to construct instantaneous QPE and QPF by taking into account the three-dimensional structure of reflectivity. First, we considered that the influence of bright band below the altitude of $3 \mathrm{~km}$ is negligible. Therefore, four altitudes of CAPPI grids, such as 0.5 , $1.0,1.5$ and $2.0 \mathrm{~km}$ are used for the input data in the RNN to capture the vertical variation of reflectivity. Preliminary studies of using three-dimensional data structures were performed by Xiao and Chandrasekar (1997) and Trafalis et al. (2002). Nevertheless, they used the same size of data grid at different altitudes without considering the influence of horizontal advection. This is because the data observed by the radar aloft is associated with rain rate at the ground with some time delay and shift in space. The influence of horizontal advection can displace precipitation from its location of measurement by radar to its location where it reaches the ground. Therefore, in order to account for the impacts of horizontal advection, larger grids of data were segmented from the original CAPPI with increasing altitude. Hence, the next step is to decide the coverage of each altitude, which is associated with the horizontal wind speed and vertical (terminal) velocity of raindrops. Before designing the size of the data grid, it is important to make sure of the time that raindrops drop to the ground from different altitudes. According to Atlas et al. (1973), we can determine the terminal velocity by using the empirical formula as follows:

$V_{\mathrm{t}}=9.65-10.3 \exp (-600 D)$

where $V_{\mathrm{t}}$ is the terminal velocity $(\mathrm{m} / \mathrm{s})$ and $D$ is the diameter of raindrop $(\mathrm{m})$.

The diameter of raindrops used in this study is quoted from Jorgensen and Willis (1982). In their research, they concluded that a single drop size is commonly smaller than $4 \mathrm{~mm}$ in a typhoon event. Accordingly, it is reasonable to assume that the maximum drop size used in this study to calculate the terminal velocity is also $4 \mathrm{~mm}$. After calculation, the terminal velocity is $8.72 \mathrm{~m} / \mathrm{s}$ (which represents the maximum velocity). Therefore, the time that the raindrops drop to the ground from the highest elevation CAPPI is about $230 \mathrm{~s}$. And this will result in a $6.9 \mathrm{~km}$ shift in the horizontal if we assume that the tangential wind speed of typhonic circulation after landing is $30 \mathrm{~m} / \mathrm{s}$. Therefore, the size of the highest elevation CAPPI grid used in this paper is $15 \times 15 \mathrm{~km}^{2}$. According to above procedure, the sizes of other elevation CAPPI grids are $11 \times 11 \mathrm{~km}^{2}, 7 \times 7 \mathrm{~km}^{2}$, and $3 \times 3 \mathrm{~km}^{2}$. Fig. 3 illustrates such a configuration of the reflectivity data. The three-dimensional structure of reflectivity was applied to RNN as the inputs and the correspond- 


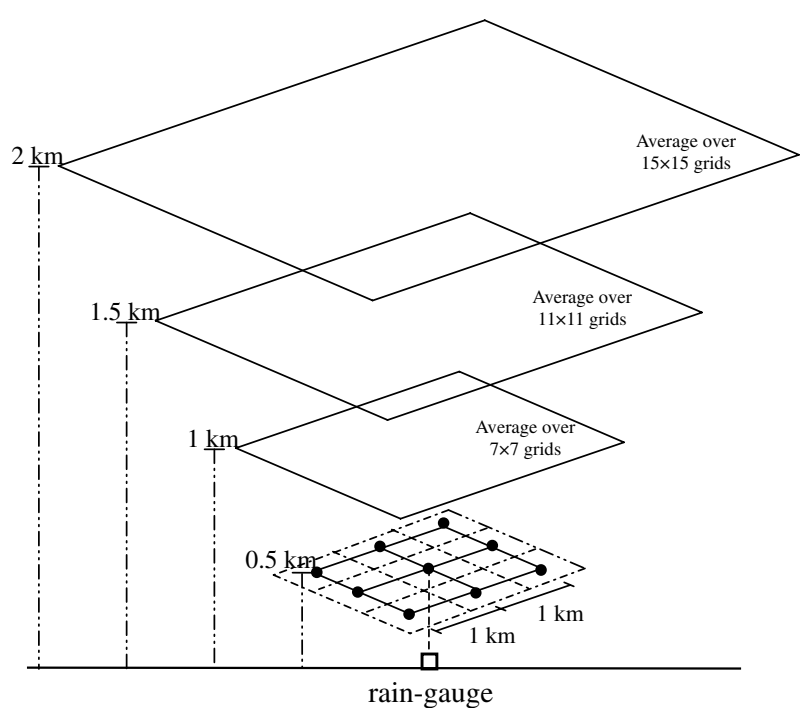

Figure 3 Three-dimensional data structure for RNN as inputs.

ing ground rain gauge data were used as the target output to construct the instantaneous QPE and one-hour-ahead QPF.

In instantaneous quantitative precipitation estimation (QPE), the input and output dimensions for RNN are 12 and 1 , respectively. The hourly rainfall is used as target output and 12 reflectivity data points are selected as model inputs. These inputs consist of nine data points over a $3 \times 3 \mathrm{~km}^{2}$ area at the $0.5 \mathrm{~km}$ elevation (as shown in Fig. 3, there are nine black points on the $3 \times 3$ grids) and three spatial mean reflectivity averaged over $7 \times 7 \mathrm{~km}^{2}, 11 \times 11 \mathrm{~km}^{2}$, and $15 \times 15 \mathrm{~km}^{2}$ grids for $1 \mathrm{~km}, 1.5 \mathrm{~km}$, and $2 \mathrm{~km}$ elevations, respectively. The concept of such input combination is that the correlation between the lowest elevation reflectivity and rainfall is more significant and robust than the other three elevations, and the variability in three higher levels is greater than the lowest level. Besides, using the spatial mean reflectivity in three higher levels could efficiently reduce the size of the input dimensions. Finally, we determine the number of hidden neurons during the calibration phase, which is combining the calibration set of all gauges, and the network output is the estimation of current rainfall.

To evaluate the properties of the developed instantaneous QPE model, we compared its predictive ability and accuracy with a power-law parametric equation, namely $Z-R$ relationship. The $Z-R$ relation used to estimate current rainfall in this study is the same as the formula used by the Central Weather Bureau (CWB), Taiwan. The form of the Z$R$ relation used by CWB, proposed by Xin et al. (1997), is shown as follows:

$Z=32.5 R^{1.65}$

where $Z$ represents the spatial mean reflectivity averaged over $3 \times 3 \mathrm{~km}^{2}$ at $0.5 \mathrm{~km}$ elevation at time $t$ (hourly), and $R$ represents the hourly rainfall accumulation at time $t$ in this study. Besides, we have additionally regressed two Z$R$ relations to evaluate the QPE from different altitudes $(0.5 \mathrm{~km}$ and $1.0 \mathrm{~km})$ in this case study. These two $Z-R$ relations are obtained utilizing least-square error method to determine the parameters ( $a$ and $b$ ) of power-law function. The optimization is made by using a sample size of 465 from all calibration sets of three rain gauges. Fig. 4(a) and (b) show the scatter of reflectivity and gauge measurements and the fitted curve to the data for QPE at 0.5 and $1.0 \mathrm{~km}$ elevation, respectively.

Three error statistics are used to assess the agreement between ground rain gauges and QPE values from both methods: relative bias, root mean square error (RMSE), and correlation of coefficient $\left(R^{2}\right)$. The relative bias is an indicator of the quality of the total estimated rainfall. The value of RMSE and $R^{2}$ can show the degree of scatter and the status of fitting between observation and forecasting. The three statistics are defined as follows:

$$
\begin{aligned}
& \text { bias }=\frac{\sum_{i=1}^{N} R_{\mathrm{est}}(i)-\sum_{i=1}^{N} R_{\mathrm{obs}}(i)}{\sum_{i=1}^{N} R_{\mathrm{obs}}(i)} \\
& \mathrm{RMSE}=\sqrt{\frac{\sum_{i=1}^{N}\left(R_{\mathrm{est}}(i)-R_{\mathrm{obs}}(i)\right)^{2}}{N}} \\
& R^{2}=\frac{\sum_{i=1}^{N}\left(R_{\mathrm{obs}}(i)-\bar{R}_{\mathrm{obs}}(i)\right)\left(R_{\mathrm{est}}(i)-\bar{R}_{\mathrm{est}}(i)\right)}{\sqrt{\sum_{i=1}^{N}\left(R_{\mathrm{obs}}(i)-\bar{R}_{\mathrm{obs}}(i)\right)^{2} \sum_{i=1}^{N}\left(R_{\mathrm{est}}(i)-\bar{R}_{\mathrm{est}}(i)\right)^{2}}}
\end{aligned}
$$

where $R_{\text {est }}$ is the estimated or forecasted value $(\mathrm{mm} / \mathrm{h})$, $R_{\text {obs }}$ is the observed value $(\mathrm{mm} / \mathrm{h})$, and $\bar{R}_{\text {obs }}$ and $\bar{R}_{\text {est }}$ are the mean of observation and estimation (or forecasting), respectively $(\mathrm{mm} / \mathrm{h})$.

Furthermore, we also constructed a one-hour-ahead forecasting network and investigated whether radar observations are useful information for RNN-based one-hourahead QPF. A simple way to do this job is to perform the same constructed RNN structure through using the reflectivity values of the next hour. The next hour reflectivity data can be estimated by using linear extrapolation technique as follows.

$x_{n}^{\prime}(t+1)=x_{n}(t)+\left[x_{n}(t)-x_{n}(t-1)\right]$

where $x_{n}^{\prime}(t+1)$ represents the hourly extrapolated value of reflectivity at time $t+1 ; x_{n}(t)$ and $x_{n}(t-1)$ represent the observed reflectivity at time $t$ and $t-1$, respectively.

Therefore, the input and output dimensions for RNN are $12\left(x_{1}^{\prime}(t+1), x_{2}^{\prime}(t+1), \ldots, x_{12}^{\prime}(t+1)\right)$ and $1(z(t+1)$ represents the hourly rainfall accumulation at time $t+1)$, respectively. In common with RNN, the reflectivity used to construct the $Z-R$ relation is also processed through the same procedure. Two $Z-R$ relations have been obtained, by least square error method, to forecast one-hour-ahead rainfall by making use of spatial mean reflectivity data averaged over $0.5 \mathrm{~km}$ and $1.0 \mathrm{~km}$ elevations and rain gauges observed pairs. Fig. 4(c) and (d) show the scatter plot and the fitted curve to the data at 0.5 and $1.0 \mathrm{~km}$ elevations, respectively.

\section{Results and discussion}

A series of typhoon events were analyzed for instantaneous QPE and QPF. By using all the radar-rain gauge data pairs from the six typhoon events, the available data were divided into calibration and two evaluation sets that consisted of 465, 144, and 144 full volume scans, respectively. A power-law $Z-R$ function was deployed to assess the performance of the proposed RNN model. The number of hidden neurons used in RNN is five; that was established in the cal- 

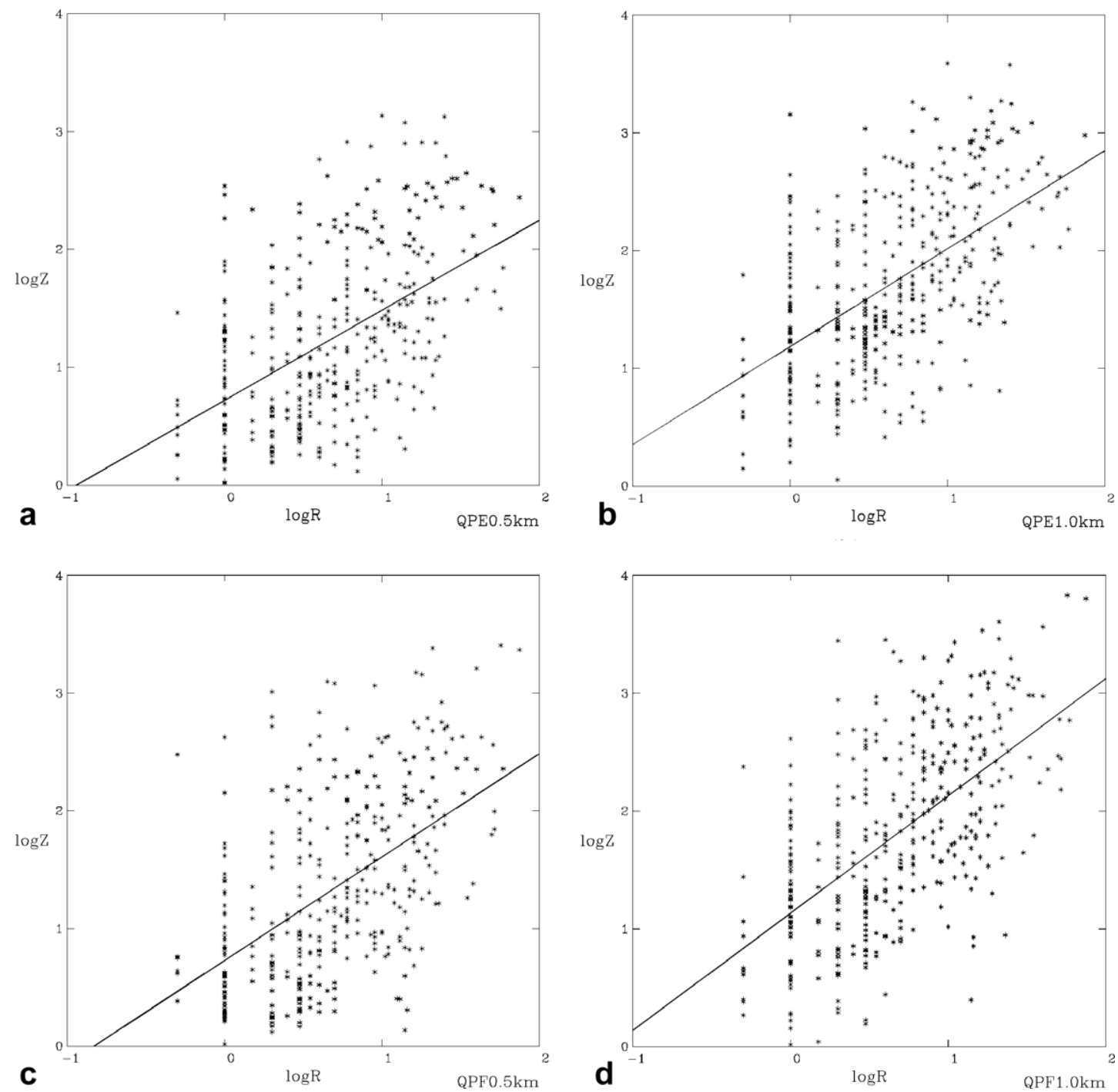

Figure 4 The scatter and the fitted curve to the data in calibration set for QPE at: (a) $0.5 \mathrm{~km}$ (b) $1.0 \mathrm{~km}$ and for QPF at (c) $0.5 \mathrm{~km}$ (d) $1.0 \mathrm{~km}$.

ibration phase, while the learning rate was determined through a trial-and-error process. Table 3 summarizes the comparative results of the above two methods for instantaneous QPE. It appears that RNN produces better hourly rainfall estimates, in terms of smaller RMSE in both evaluation phases, than those obtained from $Z-R$ relations.

Table 3 Results of the $Z-R$ relations and RNN for instantaneous QPE

\begin{tabular}{llllll}
\hline & \multicolumn{2}{l}{ Evaluation \#1 } & & \multicolumn{2}{l}{ Evaluation \#2 } \\
\cline { 2 - 3 } & RMSE $(\mathrm{mm})$ & $R^{2}$ & & RMSE $(\mathrm{mm})$ & $R^{2}$ \\
\hline$Z-R$ relation $(\mathrm{CWB})$ & 9.55 & 0.61 & 11.40 & 0.50 \\
$Z_{0.5}-R$ relation & 8.25 & 0.69 & 10.31 & 0.54 \\
$Z_{1.0}-R$ relation & 9.80 & 0.59 & 10.41 & 0.54 \\
RNN, 12 inputs & 7.12 & 0.66 & 8.81 & 0.64 \\
\hline
\end{tabular}

Because the information of total rainfall is exceedingly important in operational hydrology, rainfall accumulations obtained from both models are compared with rain gauge measurements for the entire duration of the evaluation phase. The rainfall accumulation is obtained by accumulating hourly forecasts. Performances of RNN and Z- $R$ relation for QPE in evaluation \#1 and \#2 are given in Figs. 5 and 6, respectively. It should be noticed that the results shown in these Figure are displayed in the form of every six-hour accumulation for entire event at different rain gauges. It is amazing that the results of the RNN and $Z-R$ relation are vitally different even though the $Z_{0.5}-R$ relation produces slightly better $R^{2}$ value in evaluation \#1. This is due to that the $Z-R$ relation can reflect the rainfall pattern but can't well capture the amount of rainfall. Inspection of these figures clearly indicates the differences in total rainfall estimations by these two methods. The rainfall obtained from $Z-R$ relation in both evaluation sets are extraordinarily underestimated with relative bias larger than $-45 \%$ at all three gauging stations (as shown in 

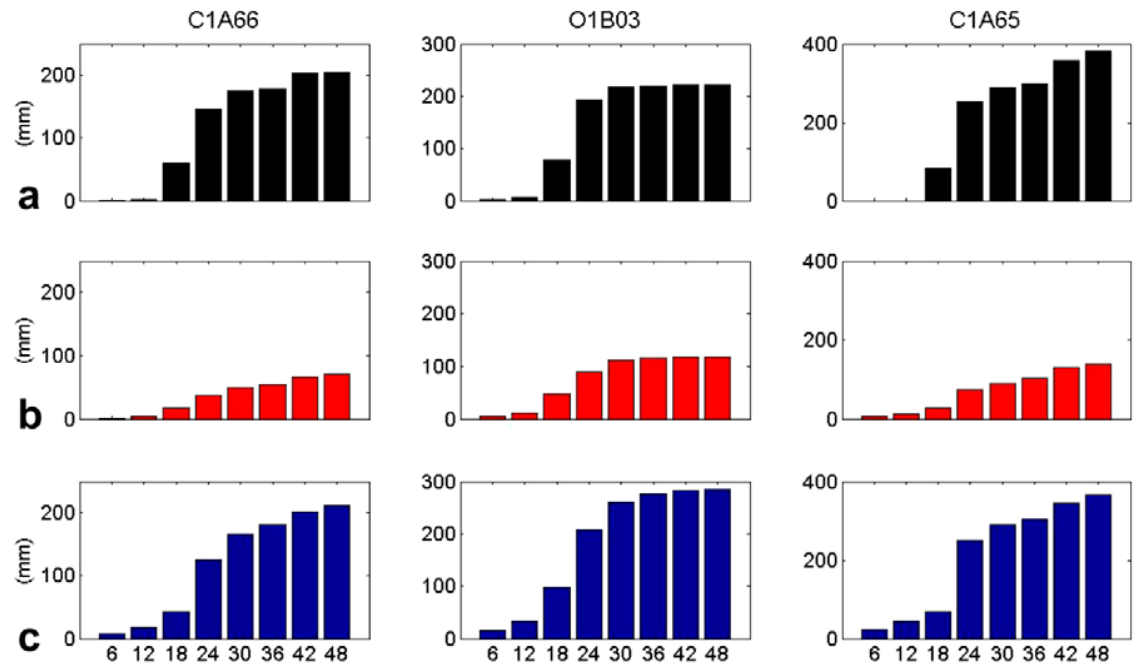

(hr)

Figure 5 Comparison of rainfall accumulations for QPE at three rain gauges in evaluation phase \#1 (a) observation, (b) $Z_{0.5}-R$ relation, (c) RNN.
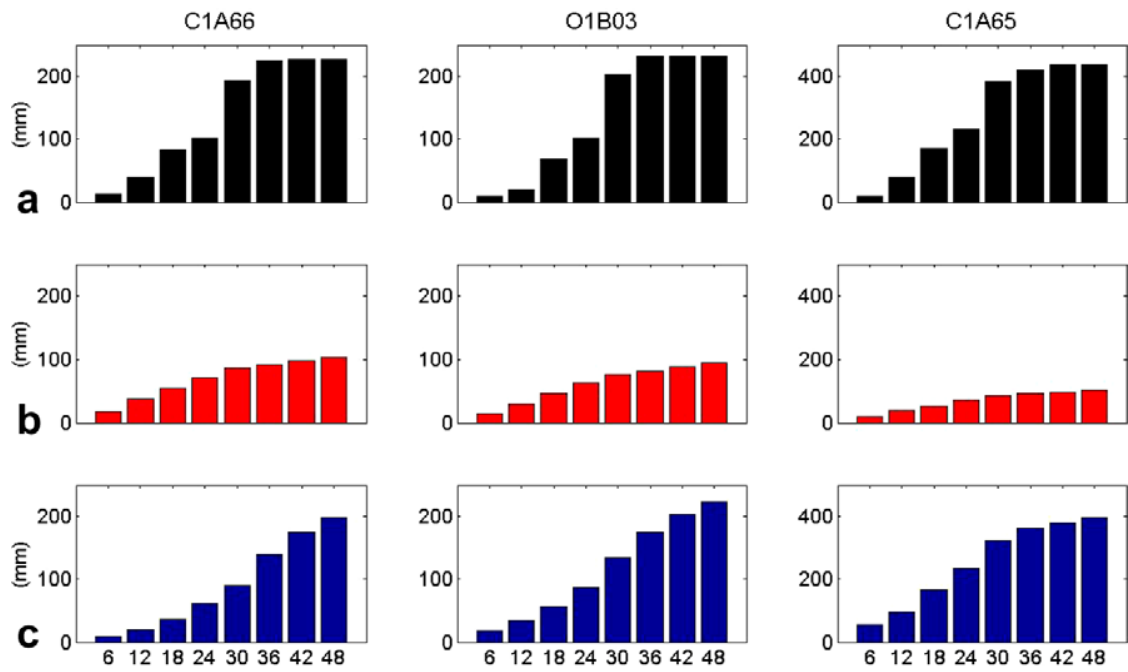

Figure 6 Comparison of rainfall accumulations for QPE at three rain gauges in evaluation phase \#2 (a) observation, (b) $Z_{0.5}-R$ relation, (c) RNN.

Table 4). Whereas, the corresponding values for RNN estimates are within $\pm 5 \%$ in evaluation \#1 except for $01 \mathrm{~B} 03$ gauging station with a little overestimate $(+28 \%)$. For evalu- ation $\# 2$, the relative bias calculated from $\mathrm{RNN}$ is $-13 \%$, $-4 \%$, and $-9 \%$ for $\mathrm{C} 1 \mathrm{~A} 66,01 \mathrm{~B} 03$, and $\mathrm{C} 1 \mathrm{~A} 65$ gauges, respectively. The results show that the RNN-based estimates of

Table 4 Comparison of 48-h rainfall accumulations $(\mathrm{mm})$ obtained from $Z-R$ relation and RNN for QPE in both evaluation sets

\begin{tabular}{|c|c|c|c|c|c|c|}
\hline & \multicolumn{3}{|c|}{ Evaluation \#1 } & \multicolumn{3}{|c|}{ Evaluation \#2 } \\
\hline & C1A66 & 01B03 & C1A65 & C1A66 & 01B03 & C1A65 \\
\hline Observation & 206 & 222 & 382 & 228 & 233 & 436 \\
\hline$Z-R$ relation & $71(-65 \%)$ & $118(-47 \%)$ & $139(-64 \%)$ & $104(-54 \%)$ & $94(-60 \%)$ & $103(-76 \%)$ \\
\hline RNN & $212(+3 \%)$ & $285(+28 \%)$ & $367(-4 \%)$ & $198(-13 \%)$ & $224(-4 \%)$ & $396(-9 \%)$ \\
\hline
\end{tabular}

*, Values within the parentheses represent relative bias. 
Table 5 Results of the $Z-R$ relations and RNN for one-hourahead QPF

\begin{tabular}{lccllll}
\hline & \multicolumn{2}{c}{ Evaluation \#1 } & & \multicolumn{2}{c}{ Evaluation \#2 } \\
\cline { 2 - 3 } & RMSE $(\mathrm{mm})$ & $R^{2}$ & & RMSE $(\mathrm{mm})$ & $R^{2}$ \\
\hline$Z_{0.5}-R$ relation & 9.86 & 0.58 & & 10.72 & 0.48 \\
$Z_{1.0}-R$ relation & 10.33 & 0.56 & & 10.98 & 0.43 \\
RNN, 12 inputs & 7.44 & 0.63 & & 9.19 & 0.56 \\
\hline
\end{tabular}

rainfall accumulations agree very well at these three gauging stations and consistently maintain the model performance in both evaluation phases.
Table 5 shows the statistical comparison of one-hourahead rainfall forecasting obtained from two $Z-R$ relations and RNN. It appears RNN has significantly better performance than the two $Z-R$ relations, where the $R^{2}$ values of RNN are 0.63 and 0.56 for evaluation sets 1 and 2, respectively. While the $R^{2}$ values of two $Z-R$ relations are varied from 0.43 to 0.58 in both evaluation sets. The values of RMSE of RNN are also much better than the $Z-R$ relations. In addition, the results listed in Figs. 7 and 8 and Table 6 indicate the observed versus one-hour-ahead forecasted rainfall by $Z-R$ relation and RNN. We can easily find that the relative bias at these three rain gauges for RNN-based QPF gives fairly accurate forecasts over every six hours, providing the total rainfall to an accuracy of $-2 \%,+18 \%$, and
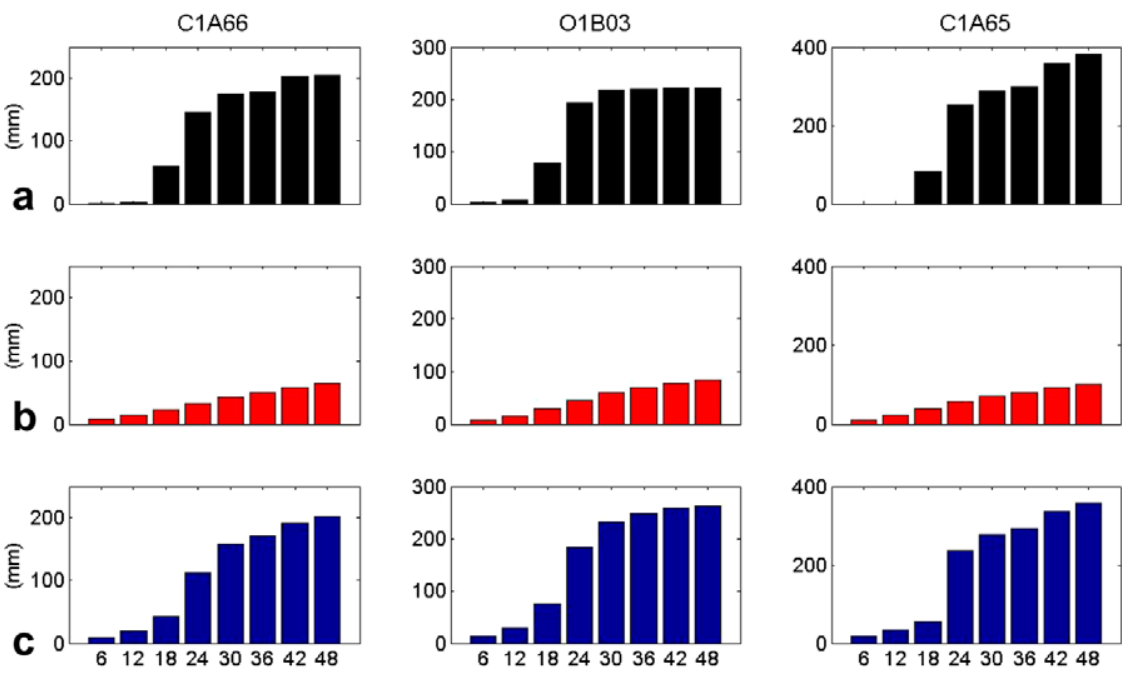

(hr)

Figure 7 Comparison of rainfall accumulations for QPF at three rain gauges in evaluation phase \#1 (a) observation, (b) $Z_{0.5}-R$ relation, (c) RNN.
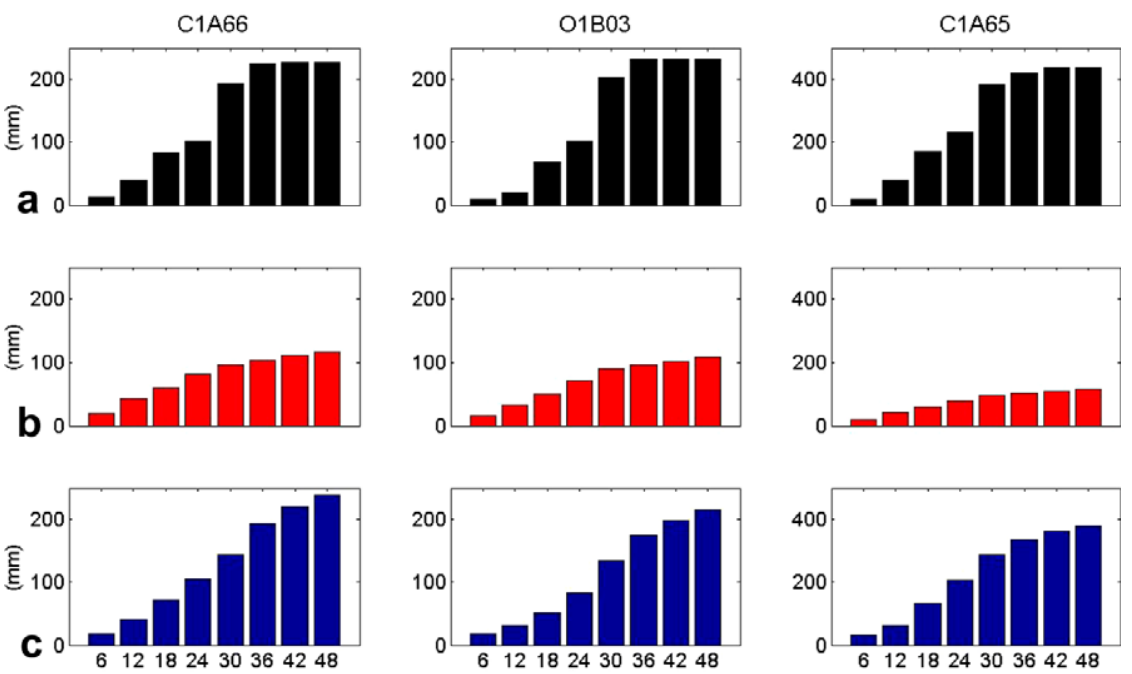

(hr)

Figure 8 Comparison of rainfall accumulations for QPF at three rain gauges in evaluation phase \#2 (a) observation, (b) $Z_{0.5}-R$ relation, (c) RNN. 
Table 6 Comparison of 48-h rainfall accumulations $(\mathrm{mm})$ obtained from $\mathrm{Z}-\mathrm{R}$ relation and RNN for QPF in both evaluation sets

\begin{tabular}{|c|c|c|c|c|c|c|}
\hline & \multicolumn{3}{|c|}{ Evaluation \#1 } & \multicolumn{3}{|c|}{ Evaluation \#2 } \\
\hline & C1A66 & 01B03 & C1A65 & C1A66 & O1B03 & C1A65 \\
\hline Observation & 206 & 222 & 382 & 228 & 233 & 436 \\
\hline$Z-R$ & $65(-68 \%)$ & $83(-63 \%)$ & $100(-74 \%)$ & $117(-49 \%)$ & $108(-54 \%)$ & $116(-73 \%)$ \\
\hline RNN & $202(-2 \%)$ & $262(+18 \%)$ & $359(-6 \%)$ & $239(+5 \%)$ & $215(-8 \%)$ & $381(-13 \%)$ \\
\hline
\end{tabular}

$-6 \%$ during typhoon Herb and $+5 \%,-8 \%$, and $-13 \%$ during typhoon Nanmadol. While, the corresponding values of the $Z-R$ relation in both evaluation sets are worse than $-49 \%$ with an extreme underestimation of $141 \mathrm{~mm}, 139 \mathrm{~mm}$, and $282 \mathrm{~mm}$ in evaluation \#1 and $111 \mathrm{~mm}, 125 \mathrm{~mm}$, and $320 \mathrm{~mm}$ in evaluation \#2 at these three rain gauges. Moreover, it can also be observed from Tables 3 and 5 that the performance of both $Z-R$ relations generated from $0.5 \mathrm{~km}$ elevation are better than that of $1.0 \mathrm{~km}$ elevation. These results also coincide with our thinking that the reflectivity of lowest elevation has better correlation with rainfall. Secondly, the performance of RNN for both QPE and QPF maintained similar accuracy. This finding might possibly be explained by the fact that the RNN model takes into account the whole radar information (three-dimensional structure) and therefore decreases the chances of mismatching fea- tures. The performances demonstrate that RNN is relatively stable and performs consistent forecasts for QPE and QPF in both evaluation sets, whereas the $Z-R$ relation could not produce good rainfall forecasting.

To summarize our results further, Figs. 9 and 10 illustrate the rainfall pattern from observations and RNN-based estimates and the corresponding error histograms at each rain gauge for instantaneous QPE and one-hour-ahead QPF, respectively. Error is defined as the difference between the forecasts and observations. Fig. 9 shows that all the forecasting errors are less than $\pm 10 \mathrm{~mm}$ in C1A66 and 01B03 gauge stations, while slightly underestimated (more than $10 \mathrm{~mm}$ ) over some periods in C1A65 gauge station. This is mainly because the rain rate rapidly intensified within one hour and the maximum rainfall occurred; the RNN could not cope with the situation due to the hourly timescale input
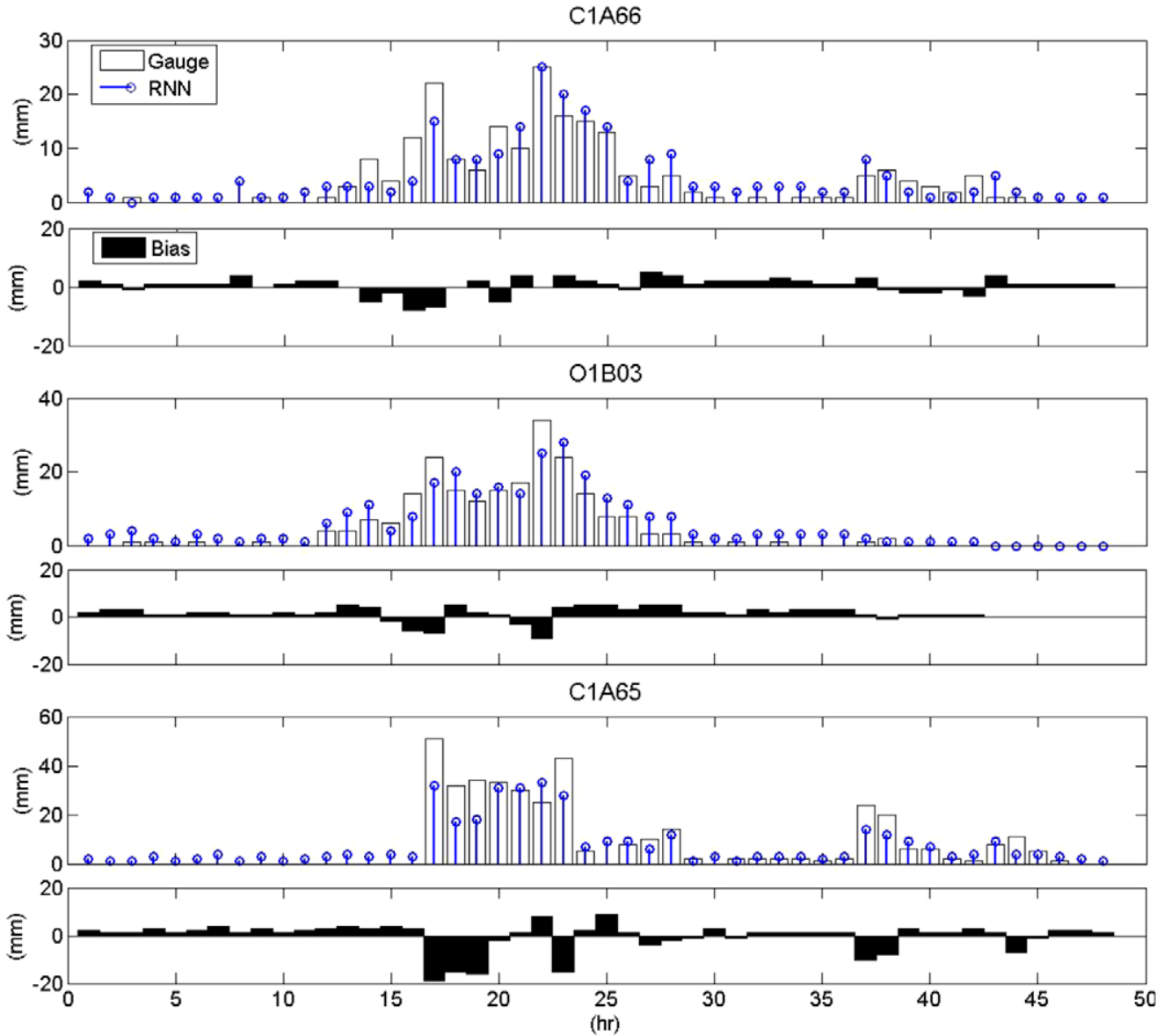

Figure 9 The rainfall pattern of measurements and RNN based estimates and related error histogram for QPE at different rain gauges in evaluation phase \#1. 

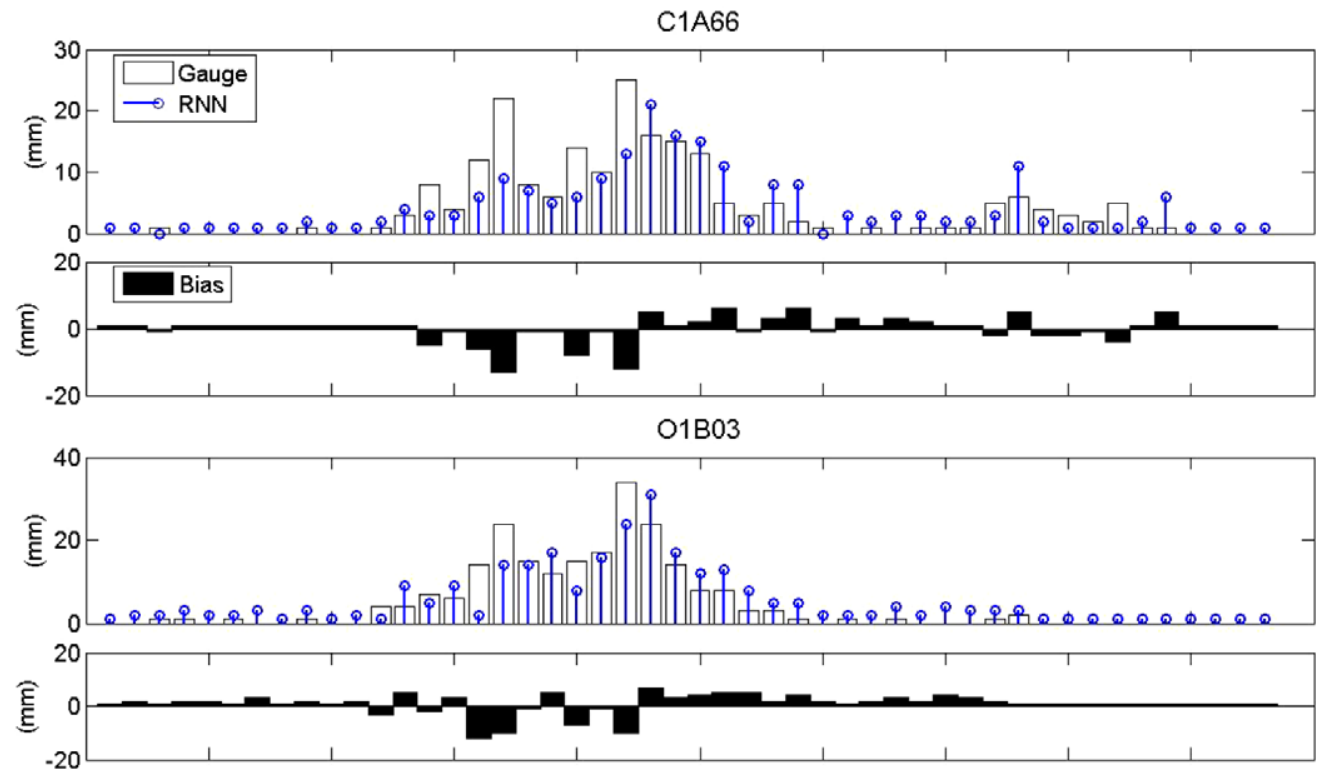

C1A65
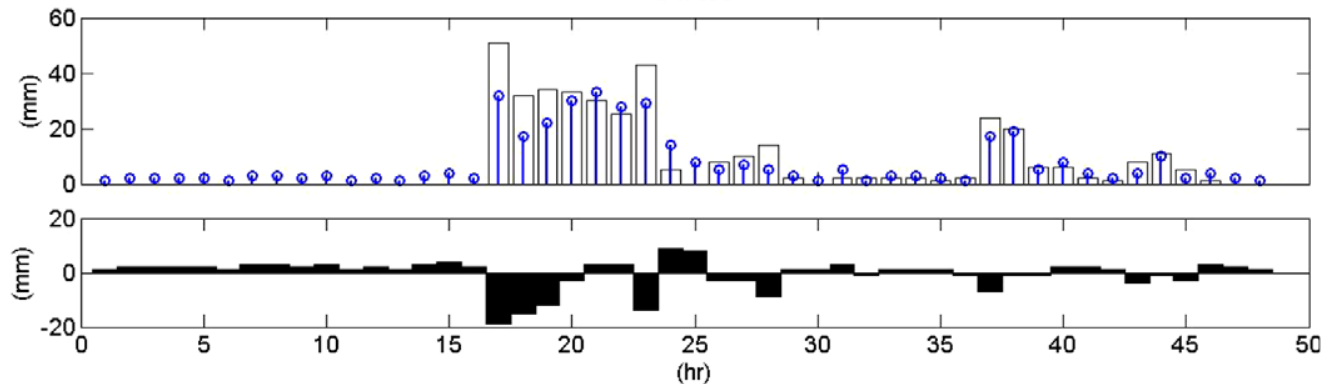

Figure 10 The rainfall pattern of measurements and RNN based estimates and related error histogram for QPF at different rain gauges in evaluation phase \#1.

data. Similarly, the results of RNN-based one-hour-ahead forecasting (Fig. 10) have the same problem where the major error occurs at the time of maximum rain rate in C1A65 gauge station. Except for that, the error values of RNNbased QPF, in general, are very small (less than $\pm 10 \mathrm{~mm}$ ) in both instantaneous estimation and one-hour-ahead forecast. One last point we would like to make is that there are only about 465 data to build and calibrate the network. In a complex system such as this, the calibration data are insufficient. It is commonly known that a neural network usually produces unacceptable forecasts if the weights have not been well trained. Nevertheless, a major characteristic of RTRL is that the algorithm has the ability to produce more accurate performance than the traditional batch leaning algorithms used in feedforward neural networks under limited data (Chiang et al., 2004). The results demonstrate the dynamic RNN with RTRL algorithm can be calibrated adequately by using a small amount of data and obtain satisfactory performance.

As far as the estimated bias is concerned, a simple $Z-R$ power-law function only uses 2 parameters to fit all the calibration data, whereas the proposed RNN fits the data utilizing 90 parameters. As shown in Fig. 4, the large variation between rain rate and reflectivity limited the simulation capability of $Z-R$ relation. Consequently, it is reasonable to believe that a simple $Z-R$ regression model could not well fit/capture the variability of such a complex system. Furthermore, it should be noticed that the data used in this study is based on typhoon events, which is usually accompanied by heavy rain within a few hours. The spatial distribution of intense rainfall makes the $Z-R$ relation even more difficult to estimate rainfall, thus resulting in a larger bias.

In addition, the error sources on reflectivity measurements might be due to the observational errors that include errors in rain gauge measurement of rain rate and errors in radar measurement of reflectivity, such as partial beam filling, attenuation by rainfall medium, and instrumental sensor problems. Sources of uncertainties in $\mathrm{R}$ and $\mathrm{Z}$ have been discussed in many previous studies. Joss and Gori (1978) examined uncertainties in data and their effect on the determined $Z-R$ relation. Campos and Zawadzki (2000) found that the $Z-R$ relation depends on instrument sensor and on the method for data analysis. However, the variability of parameters should be further studied for better understanding of the exceptional phenomenon.

\section{Concluding remarks}

A methodology of the hourly QPE and QPF using a recurrent neural network was constructed by making use of radar reflectivity. In addition, a $Z-R$ function that is commonly used to describe the reflectivity and rain rate relationship 
was used to verify the usefulness and evaluate the precision of RNN. Data from five typhoon events, including radar measurements and three ground rain gauges over the Keelung River basin, Taiwan, were investigated by using both methods to evaluate QPE and QPF. A novel technique of this study is that we used three-dimensional radar data, which take into account the terminal velocity and the horizontal advection as the dynamic ANN input.

Based on the comparisons of model performance from Z$R$ relation and RNN, the RNN demonstrates its ability to produce more accurate and robust performance than the $Z-R$ relation. The evaluation of forecasting schemes shows that the total accumulations made by RNN are relatively consistent in comparison with the $Z-R$ relation. The RNN captures the main trend for instantaneous and one hour rainfall forecasts, while the $Z-R$ relation underestimates rainfall at both QPE and QPF. The results indicate that RNN with RTRL algorithm has high practicability for QPE and QPF and appears to be a promising approach. The constructed networks depend on the diversity and completeness of the training data. Therefore the proposed methods have some limitations as their training is based solely on typhoon events and consequently could not be applied to different weather types, such as may-yu events or the monsoon.

\section{Acknowledgements}

This paper is based on partial work supported by National Science Council, R.O.C. (Grant No. NSC 93-2313-B-002006). In addition, the authors are indebted to the editor and reviewers for their valuable comments and suggestions.

\section{References}

Atlas, D., Srivastava, R.C., Sekhon, R.S., 1973. Doppler radar characteristics of precipitation at vertical incidence. Reviews in Geophysical Space Physics 11, 1-35.

Baltas, E., Mimikou, M., 1994. Short-term rainfall forecasting using radar data. Water Resources Development 10, 67-78.

Bellerby, T., Todd, M., Kniveton, D., Kidd, C., 2000. Rainfall estimation from a combination of TRMM precipitation radar and GOES multispectral satellite imagery through the use of an artificial neural network. Journal of Applied Meteorology 39, 2115-2128.

Campos, E., Zawadzki, I., 2000. Instrumental uncertainties in Z-R relations. Journal of Applied Meteorology 39, 1088-1102.

Chang, F.J., Chang, L.C., Huang, H.L., 2002. Real-time recurrent learning neural network for stream-flow forecasting. Hydrological Processes 16, 2577-2588.

Chang, L.C., Chang, F.J., Chiang, Y.M., 2004. A two-step ahead recurrent neural network for streamflow forecasting. Hydrological Processes 18, 81-92.

Chiang, Y.M., Chang, L.C., Chang, F.J., 2004. Comparison of staticfeedforward and dynamic-feedback neural networks for rainfall-runoff modeling. Journal of Hydrology 290, 297-311.

Ciach, G.J., Krajewski, W.F., 1999. Radar-rain gauge comparisons under observational uncertainties. Journal of Applied Meteorology $38,1519-1525$.

Gourley, J.J., Maddox, R.A., Howard, K.W., Burgess, D.W., 2002. An exploratory multisensor technique for quantitative estima- tion of stratiform rainfall. Journal of Hydrometeorology 3, 166180.

Grecu, M., Anagnostou, E.N., 2001. Overland precipitation estimation from TRMM passive microwave observations. Journal of Applied Meteorology 40, 1367-1380.

Grecu, M., Krajewski, W.F., 2000. A large-sample investigation of statistical procedures for radar-based short-term quantitative precipitation forecasting. Journal of Hydrology 239, 69-84.

Grimes, D.I.F., Coppola, E., Verdecchia, M., Visconti, G., 2003. A neural network approach to real-time rainfall estimation for africa using satellite data. Journal of Hydrometeorology 4, 1119-1133.

Ham, F.M., Kostanic, I., 2001. Principles of Neurocomputing for Science \& Engineering. McGraw-Hill, New York.

Hsu, K.L., Gao, X., Sorooshian, S., Gupta, H.V., 1997. Precipitation estimation from remotely sensed information using artificial neural networks. Journal of Applied Meteorology 36, 1176-1190.

Jorgensen, D.P., Willis, P.T., 1982. A Z-R relationship for hurricianes. Journal of Applied Meteorology 21, 356-366.

Joss, J., Gori, E.G., 1978. Shapes of raindrop size distributions. Journal of Applied Meteorology 17, 1054-1061.

Kidd, C., Kniveton, D.R., Todd, M.C., Bellerby, T.J., 2003. Satellite rainfall estimation using combined passive microwave and infrared algorithms. Journal of Hydrometeorology 4, 10881104.

Kuligowski, R.J., Barros, A.P., 2001. Combining IR-microwave satellite retrieval of temperature and dewpoint profiles using artificial neural networks. Journal of Applied Meteorology 40, 2051-2067.

Marshall, J.M., Palmer, W.M.K., 1948. The distribution of raindrops with size. Journal of Applied Meteorology 5, 165-166.

Miller, L.J., Mohr, C.G., Weinheimer, A., 1986. The simple rectification in Cartesian space of folded radial velocities from Doppler radar sampling. Journal of Atmospheric and Oceanic Technology 1, 162-174.

Mimikou, M.A., Baltas, E.A., 1996. Flood forecasting based on radar rainfall measurements. Journal of Water Resources Planning and Management 122, 151-156.

Morin, E., Krajewski, W.F., Goodrich, D.C., Gao, X., Sorooshian, S., 2003. Estimating rainfall intensities from weather radar data: the scale-dependency problem. Journal of Hydrometeorology 4, 782-797.

Sauvageot, H., 1992. Radar Meteorology. Artech House, 366pp.

Seo, D.J., Breidenbach, J.P., 2002. Real-time correction of spatially nonuniform bias in radar rainfall data using rain gauge measurements. Journal of Hydrometeorology 3, 93-111.

Sorooshian, S., Hsu, K.L., Gao, X., Gupta, H.V., Imam, B., Braithwaite, D., 2000. Evaluation of PERSIANN system satellitebased estimates of tropical rain. Bulletin of the American Meteorological Society 81, 2035-2046.

Trafalis, T.B., Richman, M.B., White, A., Santosa, B., 2002. Data mining techniques for improved WSR-88D rainfall estimation. Computers \& Industrial Engineering 43, 775-786.

Williams, R., Zipser, D., 1989. A learning algorithm for continually running fully recurrent neural network. Neural Computation 1 , 270-280.

Xiao, R., Chandrasekar, V., 1997. Development of a neural network based algorithm for rainfall estimation from radar observations. IEEE Transactions on Geoscience and Remote Sensing 35, 160171.

Xin, L.Y., Recuter, G., Larochelle, B., 1997. Reflectivity-rain rate relationship for convective rainshowers in Edmonton. Atmosphere-Ocean 35, 513-521. 